\title{
SPECTRAL REFINEMENT USING A NEW PROJECTION METHOD
}

\author{
REKHA P. KULKARNI ${ }^{1}$ and N. GNANESHWAR ${ }^{1}$
}

(Received 18 October, 2002; revised 29 August, 2003)

\begin{abstract}
In this paper we consider two spectral refinement schemes, elementary and double iteration, for the approximation of eigenelements of a compact operator using a new approximating operator. We show that the new method performs better than the Galerkin, projection and Sloan methods. We obtain precise orders of convergence for the approximation of eigenelements of an integral operator with a smooth kernel using either the orthogonal projection onto a spline space or the interpolatory projection at Gauss points onto a discontinuous piecewise polynomial space. We show that in the double iteration scheme the error for the eigenvalue iterates using the new method is of the order of $h^{4 r}\left(h^{3 r}\right)^{k}$, where $h$ is the mesh of the partition and $k=0,1,2, \ldots$ denotes the step of the iteration. This order of convergence is to be compared with the orders $h^{2 r}\left(h^{r}\right)^{k}$ in the Galerkin and projection methods and $h^{2 r}\left(h^{2 r}\right)^{k}$ in the Sloan method. The error in eigenvector iterates is shown to be of the order of $h^{3 r}\left(h^{3 r}\right)^{k}$ in the new method, $h^{r}\left(h^{r}\right)^{k}$ in the Galerkin and projection methods and $h^{2 r}\left(h^{2 r}\right)^{k}$ in the Sloan method. Similar improvement is observed in the case of the elementary iteration. We show that these orders of convergence are preserved in the corresponding discrete methods obtained by replacing the integration by a numerical quadrature formula. We illustrate this improvement in the order of convergence by numerical examples.
\end{abstract}

\section{Introduction}

Let $X$ be a complex Banach space and let $T$ be a bounded linear operator defined on $X$. We are interested in numerically solving the following eigenvalue problem:

$$
T \phi=\lambda \phi, \quad \lambda \in C, \quad 0 \neq \phi \in X .
$$

If $T_{n}$ is a sequence of bounded linear operators on $X$ approximating $T$, then we solve the following eigenvalue problem:

$$
T_{n} \phi_{n}=\lambda_{n} \phi_{n}
$$

\footnotetext{
${ }^{1}$ Department of Mathematics, Indian Institute of Technology, Bombay, Powai, Mumbai 400 076, India; e-mail: rpk@math.iitb.ac.in and gnanesh@math.iitb.ac.in.
}

(C) Australian Mathematical Society 2004, Serial-fee code 1446-1811/04 
The eigenelements $\lambda_{n}$ and $\phi_{n}$ provide approximations to $\lambda$ and $\phi$, respectively. If $T_{n}$ is of rank $n$, then (1.1) is equivalent to a matrix eigenvalue problem of size $n$. In order to reach the desired accuracy one may have to choose $n$ very large, which becomes expensive. Hence spectral refinement schemes such as the RayleighSchrodinger scheme, elementary iteration and double iteration are used in practice. In the refinement schemes a matrix eigenvalue problem of relatively small size is solved only once. The eigenelements $\lambda_{n}$ and $\phi_{n}$ of this small sized eigenvalue problem provide the 0 -th iterate. In order to compute the iterates $\lambda_{n}^{(k)}$ and $\phi_{n}^{(k)}$, at each step of the iteration, we need to solve only a system of linear equations of size $n$, the coefficient matrix being the same at each step. Hence it is economical to obtain the desired accuracy by computing a sufficient number of iterates in the refinement schemes instead of increasing $n$. Refinement schemes have been extensively studied in the literature (see $[1,3,6,10]$ ). The aim of this paper is to investigate some refinement schemes using a new method proposed by Kulkarni [9]. The method is applicable to a wide variety of problems and performs better than the existing methods such as the Galerkin, projection and Sloan methods.

If $T$ is a compact linear operator and $\pi_{n}$ is a sequence of bounded projections on $X$ converging to the identity operator $I$ pointwise, then in the classical Galerkin method $T_{n}$ is chosen to be $T_{n}^{G}=\pi_{n} T \pi_{n}$. In the projection method and in the iterated Galerkin method proposed by Sloan, $T_{n}$ is $T_{n}^{P}=\pi_{n} T$ and $T_{n}^{S}=T \pi_{n}$, respectively. Recently the following new approximating operator has been introduced by Kulkarni [9]:

$$
T_{n}^{M}=\pi_{n} T \pi_{n}+\pi_{n} T\left(I-\pi_{n}\right)+\left(I-\pi_{n}\right) T \pi_{n} .
$$

Since $T$ is compact, it follows that $T_{n}^{M}$ converges to $T$ in norm. The aim of this paper is to investigate elementary iteration and double iteration using this new operator $T_{n}^{M}$. Our results can be easily extended to the Rayleigh-Schrodinger scheme and other refinement schemes.

We first show that the iterates in elementary and double iteration using the new method converge faster than the corresponding iterates in the Galerkin, projection and Sloan methods. These results are applicable to an integral operator with a continuous kernel. Next we consider $T$ to be an integral operator with a smooth kernel. The projection $\pi_{n}$ is either the orthogonal projection or the interpolatory projection at Gauss points. The range of $\pi_{n}$ is a space of piecewise polynomials of order $r$ with respect to a partition of the interval with mesh $h$. For $k=0,1,2, \ldots$, let $\mu_{n}^{(k)}$ and $\psi_{n}^{(k)}$ denote the iterates in the double iteration scheme. We prove that in the new method $\left|\mu_{n}^{(k)}-\lambda\right|=O\left(h^{4 r}\left(h^{3 r}\right)^{k}\right)$ and $\left\|\psi_{n}^{(k)}-\phi\right\|=O\left(h^{3 r}\left(h^{3 r}\right)^{k}\right)$. We also show that in the Galerkin and the projection methods $\left|\mu_{n}^{(k)}-\lambda\right|=O\left(h^{2 r}\left(h^{r}\right)^{k}\right)$ and $\left\|\psi_{n}^{(k)}-\phi\right\|=O\left(h^{r}\left(h^{r}\right)^{k}\right)$, and in the Sloan method $\left|\mu_{n}^{(k)}-\lambda\right|=O\left(h^{2 r}\left(h^{2 r}\right)^{k}\right)$ and $\left\|\psi_{n}^{(k)}-\phi\right\|=O\left(h^{2 r}\left(h^{2 r}\right)^{k}\right)$. We show that even in the case of elementary iteration the new method exhibits higher orders of convergence than the Galerkin, projection 
and Sloan methods. It is to be noted that the new method is superior to the existing methods even when the kernel is only continuous.

Here is an outline of the paper. In Section 2 we describe the elementary and the double iteration schemes. In Section 3 we prove sharper estimates for the eigenvalue iterates in the elementary and the double iteration schemes. These sharper estimates, apart from giving higher orders of convergence, also provide a justification for the observed fact that in the new method and in the projection method the error in the eigenvalue iterates is smaller than the error in the corresponding eigenvector iterates. In Section 4 we prove our main results. In Theorems 4.3-4.4 we obtain orders of convergence for the iterates in the elementary iteration using the new method. In Theorem 4.5 we obtain orders of convergence for the iterates in the double iteration using the new method. In Tables 1-3 we list the orders of convergence for the iterates in the elementary and the double iteration for the new, Galerkin, projection and Sloan methods. In practice it is necessary to replace the integrals appearing in the definition of the inner product by a numerical quadrature formula. In Section 5 we show that the orders of convergence given in Tables 1-3 are still valid after taking into account the errors introduced by the numerical quadrature formula. In Section 6 we illustrate our results by numerical examples.

\section{Preliminaries}

Let $\operatorname{BL}(X)$ denote the space of all bounded linear operators on $X$ along with the operator norm. For $T \in \mathrm{BL}(X)$, let $\rho(T)$ and $\sigma(T)$ denote the resolvent set and the spectrum of $T$, respectively:

$$
\rho(T)=\left\{z \in C:(T-z I)^{-1} \in \mathrm{BL}(X)\right\} \text { and } \sigma(T)=C \backslash \rho(T) .
$$

Consider the following eigenvalue problem:

$$
T \phi=\lambda \phi, \quad \lambda \in C, \quad \phi \in X, \quad\|\phi\|=1 .
$$

Let $T_{n}$ be a sequence of operators in $\mathrm{BL}(X)$ such that $\left\|T_{n}\right\|$ is bounded, $\left\|\left(T-T_{n}\right) T\right\| \rightarrow 0$ and $\left\|\left(T-T_{n}\right) T_{n}\right\| \rightarrow 0$. Following [1] we denote the above convergence by $T_{n} \stackrel{\nu}{\rightarrow} T$.

Let $\lambda \neq 0$ be a simple eigenvalue of $T, \epsilon$ such that $0<\epsilon<\operatorname{dist}(\lambda, \sigma(T) \backslash\{\lambda \mid)$ and $\Gamma_{\epsilon}$ is the positively oriented circle with center $\lambda$ and radius $\epsilon$. Let

$$
P=-\frac{1}{2 \pi i} \int_{\Gamma_{e}}(T-z I)^{-1} d z
$$

denote the spectral projection associated with $T$ and $\lambda$. 
Since $T_{n} \stackrel{\nu}{\rightarrow} T$, there exists $n_{0}$ such that for $n \geq n_{0}, \Gamma_{\epsilon} \subset \rho\left(T_{n}\right)$ and

$$
\alpha\left(\Gamma_{\epsilon}\right)=\sup \left\{\left\|\left(T_{n}-z I\right)^{-1}\right\|: z \in \Gamma_{\epsilon}, n \geq n_{0}\right\}<\infty \text {. }
$$

Let

$$
P_{n}=-\frac{1}{2 \pi i} \int_{\Gamma_{\epsilon}}\left(T_{n}-z I\right)^{-1} d z
$$

be the spectral projection associated with $T_{n}$ and the part of the spectrum of $T_{n}$ inside $\Gamma_{\epsilon}$. Then $\left\|P_{n}\right\| \leq \alpha\left(\Gamma_{\epsilon}\right) \epsilon \leq p$, say.

For all $n$ large enough, $\sigma\left(T_{n}\right) \cap \operatorname{Int}\left(\Gamma_{\epsilon}\right)=\left\{\lambda_{n}\right\}$ and $\lambda_{n}$ is a simple eigenvalue of $T_{n}$. Let $T_{n} \phi_{n}=\lambda_{n} \phi_{n},\left\|\phi_{n}\right\|=1$. Then

$$
S_{n}=\frac{1}{2 \pi i} \int_{\Gamma_{t}} \frac{\left(T_{n}-z I\right)^{-1}}{z-\lambda_{n}} d z
$$

is the reduced resolvent associated with $T_{n}$ and $\lambda_{n}$.

Let $T_{n}^{*} \phi_{n}^{*}=\bar{\lambda}_{n} \phi_{n}^{*},\left\langle\phi_{n}, \phi_{n}^{*}\right\rangle=1$. The spectral projection $P_{n}$ is then given by

$$
P_{n} x=\left\langle x, \phi_{n}^{*}\right\rangle \phi_{n}, \quad x \in X .
$$

Thus $\left\|P_{n}\right\|=\left\|\phi_{n}^{*}\right\|$. Since $\left\|\left(P_{n}-P\right) P\right\| \rightarrow 0$, it follows that $\left\|P_{n} \phi-\phi\right\| \rightarrow 0$. Hence

$$
\left\|\left\langle\phi, \phi_{n}^{*}\right\rangle \phi_{n}\right\|=\left|\left\langle\phi, \phi_{n}^{*}\right\rangle\right| \rightarrow\|\phi\|=1
$$

and for all $n$ large enough, $1 / 2 \leq\left|\left\langle\phi, \phi_{n}^{*}\right\rangle\right|$.

We define

$$
\phi_{(n)}=\frac{\phi}{\left\langle\phi, \phi_{n}^{*}\right\rangle} .
$$

Then $\phi_{(n)}$ is an eigenvector of $T$ associated with $\lambda$ and $\left\langle\phi_{(n)}, \phi_{n}^{*}\right\rangle=1$. Note that for all $n$ large enough, $\left\|\phi_{(n)}\right\| \leq 2$. We also have $S_{n}\left(T_{n}-\lambda_{n} I\right)=\left(T_{n}-\lambda_{n} I\right) S_{n}=I-P_{n}$.

We consider the following two iterative refinement schemes (see $[1,6,10])$.

\section{Elementary or fixed point iteration}

$$
\begin{aligned}
& \lambda_{n}^{(0)}=\lambda_{n}, \quad \phi_{n}^{(0)}=\phi_{n}, \quad \text { and for } k=1,2, \ldots, \\
& \lambda_{n}^{(k)}=\left\langle T \phi_{n}^{(k-1)}, \phi_{n}^{*}\right\rangle, \\
& \phi_{n}^{(k)}=\phi_{n}^{(k-1)}+S_{n}\left(\lambda_{n}^{(k)} \phi_{n}^{(k-1)}-T \phi_{n}^{(k-1)}\right) .
\end{aligned}
$$

The following error bounds are well known (see, for example, [1]).

THEOREM 2.1. (a) If $T_{n}$ converges to $T$ in norm, then there is a positive integer $n_{1}$ such that for all $n \geq n_{1}$ and for $k=0,1,2, \ldots$

$$
\max \left(\left|\lambda_{n}^{(k)}-\lambda\right|,\left\|\phi_{n}^{(k)}-\phi_{(n)}\right\|\right\} \leq \alpha\left\|\left(T-T_{n}\right) T\right\|\left(\beta\left\|T-T_{n}\right\|\right)^{k},
$$

where $\alpha$ and $\beta$ are constants, independent of $n$ and $k$. 
(b) If $T_{n} \stackrel{\nu}{\rightarrow} T$, then there is a positive integer $n_{1}$ such that for all $n \geq n_{1}$ and for $k=0,1,2, \ldots$

$$
\begin{gathered}
\max \left\{\left|\lambda_{n}^{(2 k)}-\lambda\right|,\left|\lambda_{n}^{(2 k+1)}-\lambda\right|,\left\|\phi_{n}^{(2 k)}-\phi_{(n)}\right\|,\left\|\phi_{n}^{(2 k+1)}-\phi_{(n)}\right\|\right\} \\
\leq \alpha\left\|\left(T-T_{n}\right) T\right\|\left(\beta \max \left\{\left\|\left(T-T_{n}\right) T\right\|,\left\|\left(T-T_{n}\right) T_{n}\right\|\right\}\right)^{k},
\end{gathered}
$$

where $\alpha$ and $\beta$ are constants, independent of $n$ and $k$.

\section{Double or modified fixed point iteration}

$$
\mu_{n}^{(0)}=\lambda_{n}, \quad \psi_{n}^{(0)}=\phi_{n}
$$

and for $k=1,2, \ldots$

$$
\begin{aligned}
\lambda_{n}^{(k)} & =\left\langle T \psi_{n}^{(k-1)}, \phi_{n}^{*}\right\rangle, & \phi_{n}^{(k-1)} & =T \psi_{n}^{(k-1)} / \lambda_{n}^{(k)}, \\
\mu_{n}^{(k)} & =\left\langle T \phi_{n}^{(k-1)}, \phi_{n}^{*}\right\rangle, & \psi_{n}^{(k)} & =\phi_{n}^{(k-1)}+S_{n}\left(\mu_{n}^{(k)} \phi_{n}^{(k-1)}-T \phi_{n}^{(k-1)}\right) .
\end{aligned}
$$

We quote the following result from [1].

THEOREM 2.2. If $T_{n} \stackrel{\nu}{\rightarrow} T$, then there is a positive integer $n_{1}$ such that for all $n \geq n_{1}$ and for $k=0,1, \ldots, \max \left\{\left|\mu_{n}^{(k)}-\lambda\right|,\left\|\psi_{n}^{(k)}-\phi_{(n)}\right\|\right\} \leq\left(\beta\left\|\left(T-T_{n}\right) T\right\|\right)^{k+1}$, where $\beta$ is a constant, independent of $n$ and $k$.

\section{Modified estimates}

We prove below some sharper estimates for the error $\left|\lambda_{n}^{(k)}-\lambda\right|$ in the elementary iteration scheme and for $\left|\mu_{n}^{(k)}-\lambda\right|$ in the double iteration scheme. In numerical experiments it has often been observed that the error in the eigenvalue iterates is smaller than the error in the corresponding eigenvector iterates. Our modified estimates provide justification for this observation when we choose $T_{n}$ to be either the new finite rank operator

$$
T_{n}^{M}=\pi_{n} T \pi_{n}+\pi_{n} T\left(I-\pi_{n}\right)+\left(I-\pi_{n}\right) T \pi_{n}
$$

introduced in [9] or the approximating operator in the projection method.

Note that if $T_{n}$ converges to $T$ in norm or if $T_{n} \stackrel{\nu}{\rightarrow} T$, then for all $n$ large enough, $\left\|T-T_{n}\right\| \leq t$ and $\left\|P_{n}\right\|=\left\|\phi_{n}^{*}\right\| \leq p$.

THEOREM 3.1. Let $T_{n}$ converge to $T$ in norm, and let $\lambda_{n}^{(k)}$ and $\phi_{n}^{(k)}$ be the iterates in elementary iteration. Then there is a positive integer $n_{1}$ such that for all $n \geq n_{1}$

$$
\begin{aligned}
\left|\lambda_{n}-\lambda\right| & \leq \frac{4 p}{|\lambda|^{2}}\left\|T_{n}\left(T-T_{n}\right) T\right\|, \\
\left\|\phi_{n}-\phi_{(n)}\right\| & \leq \alpha\left\|\left(T-T_{n}\right) T\right\| .
\end{aligned}
$$


Also, for $k=1,2, \ldots$

$$
\begin{aligned}
\left|\lambda_{n}^{(k)}-\lambda\right| & \leq \frac{2 p}{|\lambda|} \alpha\left\|T_{n}\left(T-T_{n}\right)\right\|\left\|\left(T-T_{n}\right) T\right\|\left(\beta\left\|T-T_{n}\right\|\right)^{k-1}, \\
\left\|\phi_{n}^{(k)}-\phi_{(n)}\right\| & \leq \alpha\left\|\left(T-T_{n}\right) T\right\|\left(\beta\left\|T-T_{n}\right\|\right)^{k},
\end{aligned}
$$

where $\alpha$ and $\beta$ are constants, independent of $n$ and $k$.

Proof. As the estimates for $\left\|\phi_{n}^{(k)}-\phi_{(n)}\right\|$ are the same as in Theorem 2.1 (a), we only prove the estimates for $\left|\lambda_{n}^{(k)}-\lambda\right|$.

Since $T_{n}$ converges to $T$ in norm, it follows that $\lambda_{n} \rightarrow \lambda,\left.P_{n}\right|_{R(P)}: R(P) \rightarrow R\left(P_{n}\right)$ is bijective and for all large $n,\left\|\left(\left.P_{n}\right|_{R(P)}\right)^{-1}\right\| \leq 2$ (see Osborn [11]).

Hence, for all large $n,\left|\lambda_{n}\right| \geq|\lambda| / 2$. Since $\lambda_{n}$ is a simple eigenvalue of $T_{n}$, we have $T_{n} P_{n}=P_{n} T_{n}=\lambda_{n} P_{n}$. Consider

$$
\begin{aligned}
\left(\lambda-\lambda_{n}\right) \phi & =\left(\left.P_{n}\right|_{R(P)}\right)^{-1} P_{n}\left(T \phi-P_{n} T_{n} \phi\right)=\left(\left.P_{n}\right|_{R(P)}\right)^{-1} P_{n}\left(T-T_{n}\right) \phi \\
& =\frac{\left(\left.P_{n}\right|_{R(P)}\right)^{-1} P_{n} T_{n}\left(T-T_{n}\right) T \phi}{\lambda_{n} \lambda} .
\end{aligned}
$$

Hence

$$
\left|\lambda-\lambda_{n}\right| \leq \frac{\left\|\left(\left.P_{n}\right|_{R(P)}\right)^{-1}\right\|\left\|P_{n}\right\|\left\|T_{n}\left(T-T_{n}\right) T\right\|}{\left|\lambda_{n}\right||\lambda|} \leq \frac{4 p}{|\lambda|^{2}}\left\|T_{n}\left(T-T_{n}\right) T\right\|,
$$

which proves (3.1).

It can be easily seen that

$$
\lambda_{n}^{(k)}-\lambda=\left\langle\left(T-T_{n}\right)\left(\phi_{n}^{(k-1)}-\phi_{(n)}\right), \phi_{n}^{*}\right\rangle,=\frac{\left\langle T_{n}\left(T-T_{n}\right)\left(\phi_{n}^{(k-1)}-\phi_{(n)}\right), \phi_{n}^{*}\right\rangle}{\lambda_{n}} .
$$

Hence by using bounds for $\left\|\phi_{n}^{(k-1)}-\phi_{(n)}\right\|$ from Theorem 2.1 (a), we obtain

$$
\begin{aligned}
\left|\lambda_{n}^{(k)}-\lambda\right| & \leq \frac{2}{|\lambda|}\left\|P_{n}\right\|\left\|T_{n}\left(T-T_{n}\right)\right\|\left\|\phi_{n}^{(k-1)}-\phi_{(n)}\right\| \\
& \leq \frac{2 p}{|\lambda|} \alpha\left\|T_{n}\left(T-T_{n}\right)\right\|\left\|\left(T-T_{n}\right) T\right\|\left(\beta\left\|T-T_{n}\right\|\right)^{k-1}
\end{aligned}
$$

which proves (3.2).

THEOREM 3.2. Let $T_{n} \stackrel{\nu}{\rightarrow}$ T and let $\mu_{n}^{(k)}$ and $\psi_{n}^{(k)}$ be the iterates in double iteration. Then there is a positive integer $n_{1}$ such that for all $n \geq n_{1}$ and for $k=0,1,2, \ldots$

$$
\begin{aligned}
\left|\mu_{n}^{(k)}-\lambda\right| & \leq c\left\|T_{n}\left(T-T_{n}\right) T\right\|\left(\beta\left\|\left(T-T_{n}\right) T\right\|\right)^{k}, \\
\left\|\psi_{n}^{(k)}-\phi_{(n)}\right\| & \leq\left(\beta\left\|\left(T-T_{n}\right) T\right\|\right)^{k+1},
\end{aligned}
$$

where $c$ and $\beta$ are constants, independent of $n$ and $k$. 
PROOF. Let $c=\max \left\{4 p /|\lambda|^{2},\left(2 /|\lambda|^{2}+4 t p /|\lambda|^{3}\right)\right\}$. Recall that $\mu_{n}^{(0)}=\lambda_{n}^{(0)}=\lambda_{n}$. Hence the bound for $\left|\mu_{n}^{(0)}-\lambda\right|$ is proved in Theorem 3.1 , whereas the bound for $\left\|\psi_{n}^{(k)}-\phi_{(n)}\right\|$ is proved in Theorem 2.2.

Since $\lambda \neq 0$ and $\left\|\left(T-T_{n}\right) T\right\| \rightarrow 0$, choose $n_{1}$ such that for $n \geq n_{1}$,

$$
\left\|\left(T-T_{n}\right) T\right\| \leq 1 / \beta \text { and }\left\|\left(T-T_{n}\right) T\right\| \leq|\lambda| / 2 t \beta p .
$$

We have $\lambda_{n}^{(k)}-\lambda=\left\langle\left(T-T_{n}\right)\left(\psi_{n}^{(k-1)}-\phi_{(n)}\right), \phi_{n}^{*}\right\rangle$. Hence

$$
\left|\lambda_{n}^{(k)}-\lambda\right| \leq\left\|P_{n}\right\|\left\|T-T_{n}\right\|\left\|\psi_{n}^{(k-1)}-\phi_{(n)}\right\| \leq p t\left(\beta\left\|\left(T-T_{n}\right) T\right\|\right)^{k} .
$$

Thus for $n \geq n_{1}, \lambda_{n}^{(k)}-\lambda\left|\leq p t\left(\beta\left\|\left(T-T_{n}\right) T\right\|\right) \leq\right| \lambda \mid / 2$ and hence $\left|\lambda_{n}^{(k)}\right| \geq|\lambda| / 2$. Now

$$
\begin{aligned}
\mu_{n}^{(k)}-\lambda & =\left\langle\left(T-T_{n}\right)\left(\phi_{n}^{(k-1)}-\phi_{(n)}\right), \phi_{n}^{*}\right\rangle \\
& =\frac{1}{\lambda_{n}}\left\langle T_{n}\left(T-T_{n}\right) T\left(\frac{\psi_{n}^{(k-1)}-\phi_{(n)}}{\lambda_{n}^{(k)}}+\frac{\left(\lambda-\lambda_{n}^{(k)}\right) \phi_{(n)}}{\lambda \lambda_{n}^{(k)}}\right), \phi_{n}^{*}\right) .
\end{aligned}
$$

Thus

$$
\begin{aligned}
\left|\mu_{n}^{(k)}-\lambda\right| & \leq\left\|T_{n}\left(T-T_{n}\right) T\right\|\left(\frac{2}{|\lambda|^{2}}+\frac{4 t p}{|\lambda|^{3}}\right)\left(\beta\left\|\left(T-T_{n}\right) T\right\|\right)^{k} \\
& \leq c\left\|T_{n}\left(T-T_{n}\right) T\right\|\left(\beta\left\|\left(T-T_{n}\right) T\right\|\right)^{k} .
\end{aligned}
$$

This completes the proof of (3.3).

We later show that for particular choices of $T_{n},\left\|T_{n}\left(T-T_{n}\right) T\right\|$ has a higher order of convergence than $\left\|\left(T-T_{n}\right) T\right\|$. Also, $\left\|T_{n}\left(T-T_{n}\right)\right\|$ has a higher order of convergence than $\left\|T-T_{n}\right\|$. In such cases Theorems 3.1-3.2 provide sharper estimates for eigenvalue iterates than the estimates obtained from Theorems 2.1-2.2.

Let $T$ be a compact linear operator and $\left(\pi_{n}\right)$ be a sequence of bounded projections defined on $X$, that is, each $\pi_{n}$ is in $B L(X)$ and $\pi_{n}^{2}=\pi_{n}$. In the classical Galerkin, projection and Sloan methods, $T$ is approximated by $T_{n}^{G}=\pi_{n} T \pi_{n}, T_{n}^{P}=\pi_{n} T$ and $T_{n}^{S}=T \pi_{n}$, respectively. It is easy to check that $T_{n}^{G} \stackrel{\nu}{\rightarrow} T,\left\|T-T_{n}^{P}\right\| \rightarrow 0$ and $T_{n}^{S} \stackrel{\nu}{\rightarrow} T$. Since

$$
T_{n}^{M}=\pi_{n} T \pi_{n}+\pi_{n} T\left(I-\pi_{n}\right)+\left(I-\pi_{n}\right) T \pi_{n}
$$

it follows that $\left\|T-T_{n}^{M}\right\|=\left\|\left(I-\pi_{n}\right) T\left(I-\pi_{n}\right)\right\| \rightarrow 0$. Theorems $2.1,3.1$ and 3.2 are thus applicable to the above four methods.

Note that

$$
\begin{gathered}
\left\|\left(T-T_{n}^{M}\right) T\right\|=O\left(\left\|\left(I-\pi_{n}\right) T\right\|^{2}\right), \\
\left\|T-T_{n}^{P}\right\|=\left\|\left(I-\pi_{n}\right) T\right\|, \quad\left\|\left(T-T_{n}^{S}\right) T\right\|=\left\|T\left(I-\pi_{n}\right) T\right\|
\end{gathered}
$$


and

$$
\left\|\left(T-T_{n}^{G}\right) T\right\| \leq\left\|\left(I-\pi_{n}\right) T\right\|\|T\|+\left\|\pi_{n}\right\|\left\|T\left(I-\pi_{n}\right) T\right\|=O\left(\left\|\left(I-\pi_{n}\right) T\right\|\right) .
$$

The comparison of the above bounds suggests that the iterates defined by the elementary and the double iteration schemes using the new method should converge faster than the corresponding iterates obtained by using the Galerkin, projection or Sloan methods. These results are applicable to an integral operator with a continuous kernel.

In the next section we consider $T$ to be an integral operator with a smooth kernel. We choose $X_{n}=R\left(\pi_{n}\right)$ to be a space of piecewise polynomials. The projection $\pi_{n}$ is either the orthogonal projection or the interpolatory projection at Gauss points. We obtain precise orders of convergence in terms of powers of the mesh $h$ of the partition and show that the iterates using the new method converge at a much faster rate than the iterates obtained using the Galerkin, projection or Sloan methods.

\section{Main results}

Consider the integral operator

$$
(T u)(s)=\int_{0}^{1} k(s, t) u(t) d m(t), \quad s \in[0,1]
$$

where the kernel $k(\cdot, \cdot) \in C([0,1] \times[0,1])$. Then $T: L^{2}[0,1] \rightarrow L^{2}[0,1]$ or $T:$ $C[0,1] \rightarrow C[0,1]$ is a compact linear operator. Let $r \geq 0$ and let $C^{r}[0,1]$ denote the space of $r$ times continuously differentiable functions. If $k(\cdot, \cdot) \in C^{r}([0,1] \times[0,1])$, then $R(T) \subset C^{r}[0,1]$. For $u \in C^{r}[0,1], u^{(r)}$ denotes the $r$-th derivative of $u$. We set

$$
D^{i, j} k(s, t)=\frac{\partial^{i+j}}{\partial s^{i} \partial t^{j}} k(s, t), \quad s, t \in[0,1], \quad\|k\|_{r, \infty}=\sum_{i=0}^{r} \sum_{j=0}^{r}\left\|D^{i, j} k\right\|_{\infty}
$$

and $\|u\|_{r, \infty}=\sum_{i=0}^{r}\left\|u^{(i)}\right\|_{\infty}$. For $u \in L^{2}[0,1]$, we have

$$
(T u)^{(r)}(s)=\int_{0}^{1} \frac{\partial^{r}}{\partial s^{r}} k(s, t) u(t) d m(t), \quad s \in[0,1] .
$$

Hence

$$
\left\|(T u)^{(r)}\right\|_{\infty} \leq\|k\|_{r, \infty}\|u\|_{2}
$$

For any integer $n$, let

$$
0=t_{0}<t_{1}<\cdots<t_{n}=1
$$


be a partition and for $i=1, \ldots, n$, set $h_{i}=t_{i}-t_{i-1}, h=\max _{i} h_{i}$. We assume that $h \rightarrow 0$ as $n \rightarrow \infty$. Choose $r \geq 1$. Let $X_{n}=S_{r, n}^{v}$, the space of all piecewise polynomials of order $r$ (that is, of degree $\leq r-1$ ) with breakpoints at $t_{1}, \ldots, t_{n-1}$ and with $v$ continuous derivatives, $-1 \leq v \leq r-2$. Here $v=0$ corresponds to the case of continuous piecewise polynomials. If $v=-1$, there are no continuity requirements at the breakpoints, in which case we arbitrarily take $u_{n} \in X_{n}$ to be left continuous at $t_{1}, \ldots, t_{n}$ and right continuous at $t_{0}$.

4.1. Orthogonal projection Let $X=L^{2}[0,1]$. Let $($,$) and \|\cdot\|_{2}$ denote the usual inner product and the $L^{2}$ norm on $X$. Let $\pi_{n}: X \rightarrow X_{n}$ denote the orthogonal projection. When $v=-1$ or 0 it is known that, without any restriction on the partition (see Richter [12] and de Boor [4], respectively),

$$
\left\|\pi_{n}\right\|_{L^{\infty} \rightarrow L^{\infty}} \leq C .
$$

When $v \geq 1$, (4.3) remains true with the additional restriction that the partition is quasiuniform (see Douglas et al. [7]). Then $\pi_{n} u \rightarrow u$ as $n \rightarrow \infty$ for each $u \in X$. We assume that the kernel $k(\cdot, \cdot) \in C^{r}([0,1] \times[0,1])$.

In what follows we crucially use the following estimate from Chatelin [3]. For $u \in C^{r}[0,1]$,

$$
\left\|\left(I-\pi_{n}\right) u\right\|_{\infty} \leq C_{1}\left\|u^{(r)}\right\|_{\infty} h^{r}
$$

The basic idea for proving the following theorems comes from Propositions 4.1-4.4 of Kulkarni ([8]).

THEOREM 4.1. If $\pi_{n}: L^{2}[0,1] \rightarrow X_{n}$ is the orthogonal projection defined above and $T$ is an integral operator with kernel $k(\cdot, \cdot) \in C^{\prime}([0,1] \times[0,1])$, then

$$
\begin{aligned}
\left\|\left(I-\pi_{n}\right) T\right\| & =O\left(h^{r}\right), \\
\left\|\left(I-\pi_{n}\right) T\left(I-\pi_{n}\right)\right\| & =O\left(h^{2 r}\right), \\
\left\|T\left(I-\pi_{n}\right) T\right\| & =O\left(h^{2 r}\right), \\
\left\|\left(I-\pi_{n}\right) T\left(I-\pi_{n}\right) T\right\| & =O\left(h^{3 r}\right), \\
\left\|T\left(I-\pi_{n}\right) T\left(I-\pi_{n}\right)\right\| & =O\left(h^{3 r}\right), \\
\left\|T\left(I-\pi_{n}\right) T\left(I-\pi_{n}\right) T\right\| & =O\left(h^{4 r}\right)
\end{aligned}
$$

Proof. Let $u \in L^{2}[0,1]$. Since $k(\cdot, \cdot) \in C^{r}([0,1] \times[0,1]), T u \in C^{r}[0,1]$. Then by $(4.1)\left\|(T u)^{(r)}\right\|_{\infty} \leq\|k\|_{r, \infty}\|u\|_{2}$. Hence by (4.4)

$$
\left\|\left(I-\pi_{n}\right) T u\right\|_{2} \leq C_{1}\left\|(T u)^{(r)}\right\|_{\infty} h^{r} \leq C_{1}\|k\|_{r, \infty}\|u\|_{2} h^{r},
$$


which proves $(4.5)$.

Next $\left\|\left(I-\pi_{n}\right) T\left(I-\pi_{n}\right) u\right\|_{2} \leq C_{1}\left\|\left(T\left(I-\pi_{n}\right) u\right)^{(r)}\right\|_{\infty} h^{r}$. We have

$$
\left(T\left(I-\pi_{n}\right) u\right)^{(r)}(s)=\int_{0}^{l} \frac{\partial^{r}}{\partial s^{r}} k(s, t)\left(\left(I-\pi_{n}\right) u\right)(t) d m(t) .
$$

For a fixed $s \in[0,1]$ let $l_{s}(t)=\partial^{r}(k(s, t)) / \partial s^{r}, t \in[0,1]$. Then

$$
\begin{aligned}
\left|\left(T\left(I-\pi_{n}\right) u\right)^{(r)}(s)\right| & =\left|\left\langle\left(I-\pi_{n}\right)(u), \bar{l}_{s}\right\rangle\right| \\
& =\left|\left\langle u,\left(I-\pi_{n}\right) \bar{l}_{s}\right\rangle\right| \leq C_{1}\|k\|_{r, \infty}\|u\|_{2} h^{r} .
\end{aligned}
$$

Thus $\left\|\left(T\left(I-\pi_{n}\right) u\right)^{(r)}\right\|_{\infty} \leq C_{1}\|k\|_{r, \infty}\|u\|_{2} h^{r}$ and

$$
\left\|\left(I-\pi_{n}\right) T\left(I-\pi_{n}\right) u\right\|_{2} \leq\left(C_{1}\right)^{2}\|k\|_{r, \infty}\|u\|_{2} h^{2 r},
$$

which proves (4.6).

Fix $s \in[0,1]$ and let $k_{s}(t)=k(s, t), t \in[0,1]$. Consider

$$
\begin{aligned}
T\left(I-\pi_{n}\right) T u(s) & =\int_{0}^{1} k(s, t)\left(I-\pi_{n}\right)(T u)(t) d m(t) \\
& =\left\langle\left(I-\pi_{n}\right)(T u),\left(I-\pi_{n}\right) \bar{k}_{s}\right\rangle .
\end{aligned}
$$

Hence

$$
\begin{aligned}
\left\|T\left(I-\pi_{n}\right) T u\right\|_{2} & \leq\left(C_{1}\right)^{2}\left\|(T u)^{(r)}\right\|_{\infty}\left\|\left(k_{s}\right)^{(r)}\right\|_{\infty} h^{2 r} \\
& \leq\left(C_{1}\right)^{2}\left(\|k\|_{r, \infty}\right)^{2}\|u\|_{2} h^{2 r}
\end{aligned}
$$

which proves (4.7). Also,

$$
\left(T\left(I-\pi_{n}\right) T u\right)^{(r)}(s)=\int_{0}^{1} \frac{\partial^{r}}{\partial s^{r}} k(s, t)\left(I-\pi_{n}\right)(T u)(t) d m(t)
$$

Hence

$$
\left\|\left(T\left(I-\pi_{n}\right) T u\right)^{(r)}\right\|_{\infty} \leq\left(C_{1}\right)^{2}\left(\|k\|_{r, \infty}\right)^{2}\|u\|_{2} h^{2 r}
$$

Thus

$$
\begin{aligned}
\left\|\left(I-\pi_{n}\right) T\left(I-\pi_{n}\right) T u\right\|_{2} & \leq C_{1}\left\|\left(T\left(I-\pi_{n}\right) T u\right)^{(r)}\right\|_{\infty} h^{r} \\
& \leq\left(C_{1}\right)^{3}\left(\|k\|_{r, \infty}\right)^{2}\|u\|_{2} h^{3 r}
\end{aligned}
$$

which proves (4.8). We have by (4.11) and (4.12)

$$
\begin{aligned}
\left\|T\left(I-\pi_{n}\right) T\left(I-\pi_{n}\right) u\right\|_{2} & \leq\left(C_{1}\right)^{2}\left\|\left(T\left(I-\pi_{n}\right) u\right)^{(r)}\right\|_{\infty}\left\|\left(k_{s}\right)^{(r)}\right\|_{\infty} h^{2 r} \\
& \leq\left(C_{1}\right)^{3}\left(\|k\|_{r, \infty}\right)^{2}\|u\|_{2} h^{3 r}
\end{aligned}
$$


which proves (4.9).

Lastly, using (4.11) and (4.12) we obtain

$$
\begin{aligned}
\left\|T\left(I-\pi_{n}\right) T\left(I-\pi_{n}\right) T u\right\|_{2} & \leq\left(C_{1}\right)^{2}\left\|\left(T\left(I-\pi_{n}\right) T u\right)^{(r)}\right\|_{\infty}\left\|\left(k_{s}\right)^{(r)}\right\|_{\infty} h^{2 r} \\
& \leq\left(C_{1}\right)^{4}\left(\|k\|_{r, \infty}\right)^{4}\|u\|_{2} h^{4 r} .
\end{aligned}
$$

This completes the proof of (4.10).

4.2. Interpolatory projection Let $X=C[0,1]$ with the supremum norm. Choose $r \geq 1$ and we assume that $k(\cdot, \cdot) \in C^{2 r}([0,1] \times[0,1])$. Then the integral operator defined by $(4.1)$ is a compact linear operator on $C[0,1]$.

Here we choose $X_{n}=S_{r, n}^{-1}$, the space of all piecewise polynomials of order $r$ with breakpoints at $t_{1}, \ldots, t_{n-1}$. We impose no continuity conditions at the breakpoints.

Let $B_{r}=\left\{\tau_{1}, \ldots, \tau_{r}\right\}$ denote the set of $r$ Gauss points, that is, the zeros of the (Legendre) polynomial $d^{r}\left(\left(s^{2}-1\right)^{r}\right) / d s^{r}$ in the interval $[-1,1]$. Define $f_{i}:[-1,1] \rightarrow$ $\left[t_{i-1}, t_{i}\right]$ as follows:

$$
f_{i}(t)=\frac{1-t}{2} t_{i-1}+\frac{1+t}{2} t_{i}, \quad t \in[-1,1] .
$$

Let $A=\bigcup_{i=1}^{n} f_{i}\left(B_{r}\right)=\left\{\tau_{i j}=f_{i}\left(\tau_{j}\right): 1 \leq i \leq n, 1 \leq j \leq r\right\}$, the set of $n r$ Gauss points. We define $\pi_{n}: C[0,1] \rightarrow X_{n}$ by

$$
\pi_{n} u \in X_{n}, \quad\left(\pi_{n} u\right)\left(\tau_{i j}\right)=u\left(\tau_{i j}\right), \quad 1 \leq i \leq n, 1 \leq j \leq r
$$

Then $\pi_{n} u \rightarrow u$ as $n \rightarrow \infty$ for each $u \in C[0,1]$.

The following two estimates are crucially used in the proof of Theorem 4.2.

For $u \in C^{r}[0,1]$ (see Chatelin [3]),

$$
\left\|\left(I-\pi_{n}\right) u\right\|_{\infty} \leq C_{1}\left\|u^{(r)}\right\|_{\infty} h^{r} .
$$

Let $f \in C^{r}[0,1]$ and $g \in C^{2 r}[0,1]$. Then (see de Boor-Swartz [5])

$$
\left|\int_{0}^{l} f(t)\left(I-\pi_{n}\right) g(t) d t\right| \leq C_{2}\|f\|_{r, \infty}\|g\|_{2 r, \infty} h^{2 r} .
$$

Using the above two estimates the following theorem can be proved. As the proof is along the same lines as the proof of Theorem 4.1 , we just state the result.

THEOREM 4.2. If $\pi_{n}: C[0,1] \rightarrow X_{n}$ is the interpolatory projection defined above and $T$ is an integral operator with kernel $k(\cdot, \cdot) \in C^{2 r}([0,1] \times[0,1])$, then

$$
\begin{aligned}
& \left\|\left(I-\pi_{n}\right) T\right\|=O\left(h^{r}\right), \quad \quad\left\|\left(I-\pi_{n}\right) T\left(I-\pi_{n}\right)\right\|=O\left(h^{r}\right), \\
& \left\|T\left(I-\pi_{n}\right) T\right\|=O\left(h^{2 r}\right), \quad\left\|T\left(I-\pi_{n}\right) T\left(I-\pi_{n}\right)\right\|=O\left(h^{2 r}\right), \\
& \left\|\left(I-\pi_{n}\right) T\left(I-\pi_{n}\right) T\right\|=O\left(h^{3 r}\right), \quad\left\|T\left(I-\pi_{n}\right) T\left(I-\pi_{n}\right) T\right\|=O\left(h^{4 r}\right) .
\end{aligned}
$$


Note that in the interpolatory projection the estimates for $\left\|\left(I-\pi_{n}\right) T\left(I-\pi_{n}\right)\right\|$ and $\left\|T\left(I-\pi_{n}\right) T\left(I-\pi_{n}\right)\right\|$ are different than the corresponding estimates, (4.6) and (4.9), in the orthogonal projection.

4.3. Convergence rates We give below the orders of convergence for the iterates defined by the elementary iteration. We choose $T_{n}$ to be the new approximating operator

$$
T_{n}^{M}=\pi_{n} T \pi_{n}+\pi_{n} T\left(I-\pi_{n}\right)+\left(I-\pi_{n}\right) T \pi_{n}
$$

THEOREM 4.3. Let $T$ be an integral operator with a kernel $k(\cdot, \cdot) \in C^{r}([0,1] \times$ $[0,1])$ and let $\pi_{n}$ be the orthogonal projection defined in Section 4.1. Let $\lambda_{n}^{(k)}$ and $\phi_{n}^{(k)}$ be the iterates in the elementary iteration using the new method. Then for $k=0,1,2, \ldots$

$$
\begin{aligned}
\left|\lambda_{n}^{(k)}-\lambda\right| & =O\left(h^{4 r}\left(h^{2 r}\right)^{k}\right), \\
\left\|\phi_{n}^{(k)}-\phi_{(n)}\right\| & =O\left(h^{3 r}\left(h^{2 r}\right)^{k}\right) .
\end{aligned}
$$

Proof. Let $\left\|\pi_{n}\right\| \leq \gamma$. Note that by Theorem 4.1

$$
\begin{aligned}
\left\|T-T_{n}^{M}\right\| & =\left\|\left(I-\pi_{n}\right) T\left(I-\pi_{n}\right)\right\|=O\left(h^{2 r}\right), \\
\left\|\left(T-T_{n}^{M}\right) T\right\| & =\left\|\left(I-\pi_{n}\right) T\left(I-\pi_{n}\right) T\right\|=O\left(h^{3 r}\right), \\
\left\|T_{n}^{M}\left(T-T_{n}^{M}\right)\right\| & =\left\|\pi_{n} T\left(I-\pi_{n}\right) T\left(I-\pi_{n}\right)\right\| \\
& \leq \gamma\left\|T\left(I-\pi_{n}\right) T\left(I-\pi_{n}\right)\right\|=O\left(h^{3 r}\right), \\
\left\|T_{n}^{M}\left(T-T_{n}^{M}\right) T\right\| & =\left\|\pi_{n} T\left(I-\pi_{n}\right) T\left(I-\pi_{n}\right) T\right\| \\
& \leq \gamma\left\|T\left(I-\pi_{n}\right) T\left(I-\pi_{n}\right) T\right\|=O\left(h^{4 r}\right) .
\end{aligned}
$$

The proof now follows from Theorem 3.1.

Note that if we use Theorem 2.1 , then we can only say that

$$
\left|\lambda_{n}^{(k)}-\lambda\right|=O\left(h^{3 r}\left(h^{2 r}\right)^{k}\right) \text {. }
$$

THEOREM 4.4. Let $T$ be an integral operator with a kernel $k(\cdot, \cdot) \in C^{2 r}([0,1] \times$ $[0,1])$ and let $\pi_{n}$ be the interpolatory projection defined in Section 4.2. Let $\lambda_{n}^{(k)}$ and $\phi_{n}^{(k)}$ be the iterates in the elementary iteration using the new method. Then for $k=0,1, \ldots,\left|\lambda_{n}^{(k)}-\lambda\right|=O\left(h^{4 r}\left(h^{r}\right)^{k}\right)$ and $\left\|\phi_{n}^{(k)}-\phi_{(n)}\right\|=O\left(h^{3 r}\left(h^{r}\right)^{k}\right)$.

PROOF. The proof follows from Theorems 3.1 and 4.2. 
We now consider the double iteration. The projection $\pi_{n}$ is chosen to be either the orthogonal projection described in Section 4.1 or the interpolatory projection described in Section 4.2. The operator $T$ is chosen to be an integral operator with the kernel $r$ times continuously differentiable in the case of the orthogonal projection or $2 r$ times continuously differentiable in the case of the interpolatory projection. The operator $T_{n}$ is chosen to be

$$
T_{n}^{M}=\pi_{n} T \pi_{n}+\pi_{n} T\left(I-\pi_{n}\right)+\left(I-\pi_{n}\right) T \pi_{n} .
$$

THEOREM 4.5. Let $\mu_{n}^{(k)}$ and $\psi_{n}^{(k)}$ be the iterates in the double iteration. Then for $k=0,1, \ldots,\left|\mu_{n}^{(k)}-\lambda\right|=O\left(h^{4 r}\left(h^{3 r}\right)^{k}\right)$ and $\left\|\psi_{n}^{(k)}-\phi_{(n)}\right\|=O\left(h^{3 r}\left(h^{3 r}\right)^{k}\right)$.

PROOF. The proof follows by combining the results of Theorems 3.2, 4.1 and 4.2.

In a similar manner we can obtain orders of convergence for the iterates obtained by using the Galerkin, projection and Sloan methods. We skip the proofs and just list the orders of convergence for the eigenelement iterates in the elementary and the double iteration using these three methods. It is to be noted that the eigenvalue iterates in the projection method and the Sloan method are the same.

In Table 1 we give orders of convergence for the iterates in the double iteration. The projection operator $\pi_{n}$ is either the orthogonal or the interpolatory projection. In Tables 2 and 3 we list the orders of convergence for the elementary iteration with the orthogonal and the interpolatory projections, respectively. Note that $k$ denotes the number of iterates and takes values $0,1,2, \ldots$.

TABLE 1. Orders of convergence: double iteration, orthogonal or interpolatory projection.

\begin{tabular}{|l|l|l|}
\hline Method & $\left|\mu_{n}^{(k)}-\lambda\right|$ & $\left\|\psi_{n}^{(k)}-\phi_{(n)}\right\|$ \\
\hline new & $h^{4 r}\left(h^{3 r}\right)^{k}$ & $h^{3 r}\left(h^{3 r}\right)^{k}$ \\
\hline Galerkin & $h^{2 r}\left(h^{r}\right)^{k}$ & $h^{r}\left(h^{r}\right)^{k}$ \\
\hline projection & $h^{2 r}\left(h^{2 r}\right)^{k}$ & $h^{r}\left(h^{r}\right)^{k}$ \\
\hline Sloan & $h^{2 r}\left(h^{2 r}\right)^{k}$ & $h^{2 r}\left(h^{2 r}\right)^{k}$ \\
\hline
\end{tabular}

TABLE 2. Orders of convergence: elementary iteration, orthogonal projection.

\begin{tabular}{|l|l|l|}
\hline Method & $\left|\lambda_{n}^{(k)}-\lambda\right|$ & $\left\|\phi_{n}^{(k)}-\phi_{(n)}\right\|$ \\
\hline new & $h^{4 r}\left(h^{2 r}\right)^{k}$ & $h^{3 r}\left(h^{2 r}\right)^{k}$ \\
\hline Galerkin & $h^{2 r}$ & $h^{r}: k=0$ \\
\hline & $h^{r}\left(h^{r}\right)^{k}$ & $h^{r}\left(h^{r}\right)^{k}: k \geq 1$ \\
\hline projection & $h^{2 r}\left(h^{r}\right)^{k}$ & $h^{r}\left(h^{r}\right)^{k}$ \\
\hline Sloan & $h^{2 r}\left(h^{r}\right)^{k}$ & $h^{2 r}\left(h^{r}\right)^{k}$ \\
\hline
\end{tabular}


TABLE 3. Orders of convergence: elementary iteration, interpolatory projection.

\begin{tabular}{|l|l|l|}
\hline Method & $\left|\lambda_{n}^{(k)}-\lambda\right|$ & $\left\|\phi_{n}^{(k)}-\phi_{(n)}\right\|$ \\
\hline new & $h^{4 r}\left(h^{r}\right)^{k}$ & $h^{3 r}\left(h^{r}\right)^{k}$ \\
\hline Galerkin & $h^{2 r}$ & $h^{r}: k=0$ \\
& $h^{r}\left(h^{r}\right)^{(k-1) / 2}$ & $h^{r}\left(h^{r}\right)^{(k-1) / 2}: k$ odd, $k \geq 1$ \\
& $h^{r}\left(h^{r}\right)^{k / 2}$ & $h^{r}\left(h^{r}\right)^{k / 2}: k$ even, $k \geq 2$ \\
\hline projection & $h^{2 r}\left(h^{r}\right)^{k}$ & $h^{r}\left(h^{r}\right)^{k}$ \\
\hline Sloan & $h^{2 r}\left(h^{r}\right)^{k}$ & $h^{2 r}\left(h^{r}\right)^{k}: k$ even \\
& & $h^{2 r}\left(h^{r}\right)^{k-1}: k$ odd \\
\hline
\end{tabular}

REMARK. It may be seen from Table 1 that the eigenvalue as well as eigenvector iterates in the double iteration using the new method have higher orders of convergence than the Galerkin, projection or Sloan methods. The iterates in the Sloan method converge faster than the iterates in the Galerkin method. The eigenvector iterates in the Sloan method converge faster than the corresponding iterates in the projection method. At each step of the iteration the improvement is of the order of $h^{3 r}$ in the new method, of the order of $h^{2 r}$ in the Sloan method, and of the order of $h^{r}$ in the Galerkin method. In the projection method the improvement for eigenvector iterates is of the order of $h^{r}$.

From Table 2 we observe that even in the case of elementary iteration with the orthogonal projection, the new method is superior to the Galerkin, projection and Sloan methods. Here at each step of the iteration the improvement is of the order of $h^{2 r}$ in the new method and of the order of $h^{r}$ in the Galerkin, projection and Sloan methods.

In the case of the elementary iteration with the interpolatory projection, we see from Table 3 that the iterates in the new method are still more accurate than the corresponding iterates in the Galerkin, projection and Sloan methods. But the improvement at each step of the iteration is of the order of $h^{r}$ as in the case of the projection method. In the Galerkin and Sloan methods the improvement will be respectively $h^{r}$ and $h^{2 r}$ after every two steps.

We also note that in the new method the double iteration is clearly superior to the elementary iteration. In the case of the orthogonal projection the improvement, per step of the iteration, is from $h^{2 r}$ in the elementary iteration to $h^{3 r}$ in the double iteration. In the case of the interpolatory projection this improvement is from $h^{r}$ to $h^{3 r}$ per step of the iteration.

Note that in both the elementary and the double iteration, using the new or the projection method, the eigenvalue iterates have a higher order of convergence than the corresponding eigenvector iterates.

In the new method and in the Galerkin method the elementary iteration with the 
orthogonal projection has a higher order of convergence than the elementary iteration with the interpolatory projection.

In Section 6 we illustrate the above observations by numerical examples.

Note that the dimension of the range of $\pi_{n}$ is $n r$. Then the rank of $T_{n}^{G}, T_{n}^{P}$ and $T_{n}^{S}$ is $n r$, while the rank of the new operator $T_{n}^{M}$ is $2 n r$. Thus in the new method we need to solve an eigenvalue problem double the size of the eigenvalue problem in the Galerkin, projection or Sloan methods. But as the order of convergence is improved, as given in Tables $1-3$, it is still economical to use the new method. Also, note that for double iteration, in addition to the computations in the elementary iteration, we need to compute $\lambda_{n}^{(k)}=\left\langle T \psi_{n}^{(k-1)}, \phi_{n}^{*}\right\rangle$ and $T \psi_{n}^{(k-1)} / \lambda_{n}^{(k)}$.

\section{Discrete methods}

It is to be noted that, in practice, while computing the iterates in the refinement schemes, we need to replace the integrals appearing in the integral operator $T$ and in the inner product by a numerical quadrature formula. In this section we show that if we choose the numerical quadrature formula appropriately, then the orders of convergence given in Tables $1-3$ are still valid. We first consider the case of the orthogonal projection.

Let $X=L^{2}[0,1]$. For $r \geq 1$, let $T$ be an integral operator with kernel $k(\cdot, \cdot) \in$ $C^{2 r}([0,1] \times[0,1])$ and let $X_{n}=S_{r, n}^{-1}$. The dimension of $X_{n}$ is $N=n r$. Let $\pi_{n}: L^{2}[0,1] \rightarrow X_{n}$ be the orthogonal projection. If $\left\{e_{1}, e_{2}, \ldots, e_{N}\right\}$ is an orthogonal basis for $X_{n}$, then

$$
\pi_{n} f=\sum_{j=1}^{N}\left\langle f, e_{j}\right) e_{j}, \quad f \in X
$$

Consider a fine partition $0=s_{0}<s_{1}<\cdots<s_{m}=1$ of $[0,1]$ with norm $\tilde{h}=\max \left\{s_{i}-s_{i-1}: i=1, \ldots, m\right\}$. We assume that the above partition is a refinement of the partition (4.2) defined in Section 4 so that $m>n$ and $\tilde{h}<h$. Let $g_{i}:[-1,1] \rightarrow$ $\left[s_{i-1}, s_{i}\right]$ be the affine map defined as

$$
g_{i}(t)=\frac{1-t}{2} s_{i-1}+\frac{1+t}{2} s_{i}, \quad t \in[-1,1] .
$$

Let $\left\{\eta_{i j}=g_{i}\left(\tau_{j}\right): 1 \leq i \leq m, 1 \leq j \leq r\right\}=\left\{\xi_{p}: 1 \leq p \leq R=m r\right\}$ be the set of $m r$ Gauss points. Consider the composite Gauss rule with $r$ points given by

$$
\sum_{p=1}^{R} w_{p} f\left(\xi_{p}\right) \simeq \int_{0}^{1} f(t) d t .
$$


If $f \in C^{2 r}[0,1]$, then

$$
\left|\int_{0}^{1} f(t) d t-\sum_{p=1}^{R} w_{p} f\left(\xi_{p}\right)\right| \leq C_{l}\left\|f^{(2 r)}\right\|_{\infty}(\tilde{h})^{2 r},
$$

where $C_{1}$ is a constant independent of $\tilde{h}$.

We define

$$
\left(T_{R} u\right)(s)=\sum_{p=1}^{R} w_{p} k\left(s, \xi_{p}\right) u\left(\xi_{p}\right), \quad s \in[0,1]
$$

Using (5.1), it can be easily checked that $\left\|\left(T-T_{R}\right) T\right\|=O\left(\tilde{h}^{2 r}\right)$. It is well known that if $\lambda$ is a simple eigenvalue of $T$ and $\lambda^{R}$ is the eigenvalue of $T_{R}$ approximating $\lambda$, then

$$
\left|\lambda-\lambda^{R}\right| \leq C_{2}\left\|\left(T-T_{R}\right) T\right\|=O\left(\tilde{h}^{2 r}\right)
$$

Similarly the error in the eigenvector approximation is of the order of $\tilde{h}^{2 r}$.

Following Atkinson and Bogomolny [2] we define the discrete inner product as

$$
\langle f, g\rangle_{R}=\sum_{p=1}^{R} w_{p} f\left(\xi_{p}\right) g\left(\xi_{p}\right), \quad \text { for } f, g \text { in } C[0,1] .
$$

The operator $Q_{n}: C[0,1] \rightarrow X_{n}$ is defined by

$$
Q_{n} f=\sum_{j=1}^{N}\left\langle f, e_{j}\right\rangle_{R} e_{j}
$$

We quote the following results from [2]:

$$
\left\langle Q_{n} f, g\right\rangle_{R}=\left\langle f, Q_{n} g\right\rangle_{R}, \quad f, g \in C[0,1],
$$

$\left\|Q_{n}\right\| \leq q$, a constant independent of $n$ and if $f \in C^{r}[0,1]$, then

$$
\left\|f-Q_{n} f\right\|_{\infty} \leq C_{4}\left\|f^{(r)}\right\|_{\infty} h^{r}
$$

With the above notation, a discrete version of the new approximating operator is defined as

$$
T_{n}^{D}=Q_{n} T_{R} Q_{n}+Q_{n} T_{R}\left(I-Q_{n}\right)+\left(I-Q_{n}\right) T_{R} Q_{n}
$$

Thus the integral operator $T$ is replaced by $T_{R}$, the projection $\pi_{n}$ is replaced by $Q_{n}$ and we approximate eigenelements of $T_{R}$ by $T_{n}^{D}$.

We state below a result which is similar to Theorem 4.1. All the operators in the following theorem are defined on $L^{\infty}[0,1]$. 
THEOREM 5.1. If $T$ is an integral operator with kernel $k(\cdot, \cdot) \in C^{r}([0,1] \times[0,1])$, then

$$
\begin{aligned}
& \left\|\left(I-Q_{n}\right) T_{R}\right\|=O\left(h^{r}\right), \quad \quad\left\|\left(I-Q_{n}\right) T_{R}\left(I-Q_{n}\right)\right\|=O\left(h^{2 r}\right), \\
& \left\|T_{R}\left(I-Q_{n}\right) T_{R}\right\|=O\left(h^{2 r}\right), \quad\left\|\left(I-Q_{n}\right) T_{R}\left(I-Q_{n}\right) T_{R}\right\|=O\left(h^{3 r}\right), \\
& \left\|T_{R}\left(I-Q_{n}\right) T_{R}\left(I-Q_{n}\right)\right\|=O\left(h^{3 r}\right), \quad\left\|T_{R}\left(I-Q_{n}\right) T_{R}\left(I-Q_{n}\right) T_{R}\right\|=O\left(h^{4 r}\right) \text {. }
\end{aligned}
$$

The following error estimates follow from the above result. Let $\phi_{(n)}^{R}$ be an appropriately normalised eigenvector of $T_{R}$ associated with the eigenvalue $\lambda^{R}$.

THEOREM 5.2. Let $\tilde{\lambda}_{n}^{(k)}$ and $\tilde{\phi}_{n}^{(k)}$ be the iterates in the elementary iteration obtained by using using $T_{n}^{D}$. Then for $k=0,1,2, \ldots$

$$
\begin{aligned}
\left|\tilde{\lambda}_{n}^{(k)}-\lambda^{R}\right| & =O\left(h^{4 r}\left(h^{2 r}\right)^{k}\right), \\
\left\|\tilde{\phi}_{n}^{(k)}-\phi_{(n)}^{R}\right\| & =O\left(h^{3 r}\left(h^{2 r}\right)^{k}\right) .
\end{aligned}
$$

THEOREM 5.3. Let $\tilde{\mu}_{n}^{(k)}$ and $\tilde{\psi}_{n}^{(k)}$ be the iterates in the double iteration obtained by using $T_{n}^{D}$. Then for $k=0,1,2, \ldots$

$$
\left|\tilde{\mu}_{n}^{(k)}-\lambda^{R}\right|=O\left(h^{4 r}\left(h^{3 r}\right)^{k}\right) \text { and }\left\|\tilde{\psi}_{n}^{(k)}-\phi_{(n)}^{R}\right\|=O\left(h^{3 r}\left(h^{3 r}\right)^{k}\right) .
$$

Also, it can be proved that the orders of convergence given in Tables 1-3 are valid for discrete versions of the Galerkin, projection and Sloan methods.

REMARK. From the estimates (5.2) and (5.3) we get

$$
\left|\tilde{\lambda}_{n}^{(k)}-\lambda\right|=O\left(\max \left\{\tilde{h}^{2 r}, h^{4 r}\left(h^{2 r}\right)^{k}\right\}\right)
$$

Thus the maximum order of convergence that could be attained by the iterates $\tilde{\lambda}_{n}^{(k)}$ is $\tilde{h}^{2 r}$.

In the case of uniform partitions, we have $h=1 / n$ and $\tilde{h}=1 / m$. If $m=n^{p}$, then $\tilde{h}=h^{p}$ and for $k=(2 p-4) / 2$, the $k$-th iterate $\tilde{\lambda}_{n}^{(k)}$ has the same order of convergence as that of $\lambda^{R}$. If $p=2$, that is, $m=n^{2}$, then the zero-th iterate $\tilde{\lambda}_{n}^{(0)}$ itself has the same order of convergence as that of $\lambda^{R}$. Thus the choice of $R$ is determined by the accuracy which we need and the value of $R$ determines the number of iterations. It is to be noted that $\lambda^{R}$ is obtained by solving an eigenvalue problem of size $n^{p} r$, whereas $\tilde{\lambda}_{n}^{(k)}$ is obtained by solving an eigenvalue problem of size $2 n r$ and a system of equations of size $n \boldsymbol{r}$ for each iterate. Similar considerations apply to eigenvector approximation, double iteration and various methods. 
In the case of interpolatory projection, the discrete version of the new approximating operator is defined as

$$
T_{n}^{D}=\pi_{n} T_{R} \pi_{n}+\pi_{n} T_{R}\left(I-\pi_{n}\right)+\left(I-\pi_{n}\right) T_{R} \pi_{n}
$$

where $\pi_{n}$ is the interpolatory projection at $n r$ Gauss points as defined in Section 4.2.

The following result can be proved easily.

THEOREM 5.4. If kernel $k(\cdot, \cdot) \in C^{2 r}([0,1] \times[0,1])$, then

$$
\begin{aligned}
\left\|\left(I-\pi_{n}\right) T_{R}\right\| & =O\left(h^{r}\right), & \left\|T_{R}\left(I-\pi_{n}\right) T_{R}\right\| & =O\left(h^{2 r}\right), \\
\left\|\left(I-\pi_{n}\right) T_{R}\left(I-\pi_{n}\right) T_{R}\right\| & =O\left(h^{3 r}\right), & \left\|T_{R}\left(I-\pi_{n}\right) T_{R}\left(I-\pi_{n}\right) T_{R}\right\| & =O\left(h^{4 r}\right) .
\end{aligned}
$$

Using the above results it can be proved that the orders of convergence given in Tables 1-3 are valid for discrete versions of various operators.

\section{Numerical results}

We consider the integral operator $T$ given by

$$
(T x)(s)=\int_{0}^{1} k(s, t) x(t) d m(t), \quad s \in[0,1],
$$

where $k(s, t)=\exp (s t)$ or the Green's kernel

$$
k(s, t)= \begin{cases}s(1-t) & \text { if } 0 \leq s \leq t \leq 1 \\ t(1-s) & \text { if } 0 \leq t \leq s \leq 1\end{cases}
$$

Orthogonal projection Let $X_{n}$ be the space of piecewise constant functions $(r=1)$ with respect to the equidistant partition

$$
0<\frac{1}{n}<\frac{2}{n}<\cdots<\frac{n}{n}=1
$$

and $\pi_{n}: L^{2}[0,1] \rightarrow X_{n}$ be the orthogonal projection. Let $n=2$ and $m=R=2^{9}$. We choose $w_{p}=1 / R, \xi_{p}=(2 p-1) / R, p=1, \ldots, R$, which are obtained from the composite midpoint quadrature rule.

Let $\lambda^{R}$ denote the largest eigenvalue of $T_{R}$ and $\phi^{R}=\tilde{\phi}_{(n)}^{R}$ be the associated eigenvector, appropriately normalised. The results for the iterates in the elementary and the double iteration for various methods are given in Tables 4-7. The * indicates that the error is less than or equal to $10^{-16}$. 
TABLE 4. New method, orthogonal projection, $k(s, t)=\exp (s t)$.

\begin{tabular}{|c|c|c|c|c|}
\hline$k$ & $\left|\lambda^{R}-\lambda_{n}^{(k)}\right|$ & $\left\|\phi^{R}-\phi_{n}^{(k)}\right\|$ & $\left|\lambda^{R}-\mu_{n}^{(k)}\right|$ & $\left\|\phi^{R}-\psi_{n}^{(k)}\right\|$ \\
\hline 0 & $1.7 \times 10^{-5}$ & $3.0 \times 10^{-4}$ & $1.7 \times 10^{-5}$ & $3.0 \times 10^{-4}$ \\
\hline 1 & $1.2 \times 10^{-7}$ & $2.1 \times 10^{-6}$ & $7.2 \times 10^{-10}$ & $1.2 \times 10^{-8}$ \\
\hline 2 & $8.9 \times 10^{-10}$ & $1.5 \times 10^{-8}$ & $2.6 \times 10^{-14}$ & $5.0 \times 10^{-13}$ \\
\hline 3 & $6.4 \times 10^{-12}$ & $1.1 \times 10^{-10}$ & $*$ & $*$ \\
\hline
\end{tabular}

TABLE 5. Galerkin method, orthogonal projection, $k(s, t)=\exp (s t)$.

\begin{tabular}{|c|c|c|c|c|}
\hline$k$ & $\left|\lambda^{R}-\lambda_{n}^{(k)}\right|$ & $\left\|\phi^{R}-\phi_{n}^{(k)}\right\|$ & $\left|\lambda^{R}-\mu_{n}^{(k)}\right|$ & $\left\|\phi^{R}-\psi_{n}^{(k)}\right\|$ \\
\hline 0 & $2.4 \times 10^{-3}$ & $4.3 \times 10^{-2}$ & $2.4 \times 10^{-3}$ & $4.3 \times 10^{-2}$ \\
\hline 1 & $2.4 \times 10^{-3}$ & $9.3 \times 10^{-4}$ & $1.4 \times 10^{-5}$ & $1.9 \times 10^{-5}$ \\
\hline 2 & $1.4 \times 10^{-5}$ & $1.9 \times 10^{-5}$ & $9.5 \times 10^{-9}$ & $1.2 \times 10^{-8}$ \\
\hline 3 & $1.0 \times 10^{-6}$ & $4.1 \times 10^{-7}$ & $6.5 \times 10^{-12}$ & $8.7 \times 10^{-12}$ \\
\hline
\end{tabular}

TABLE 6. Projection method, orthogonal projection, $k(s, t)=\exp (s t)$.

\begin{tabular}{|c|c|c|c|c|}
\hline$k$ & $\left|\lambda^{R}-\lambda_{n}^{(k)}\right|$ & $\left\|\phi^{R}-\phi_{n}^{(k)}\right\|$ & $\left|\lambda^{R}-\mu_{n}^{(k)}\right|$ & $\left\|\phi^{R}-\psi_{n}^{(k)}\right\|$ \\
\hline 0 & $2.4 \times 10^{-3}$ & $4.3 \times 10^{-2}$ & $2.4 \times 10^{-3}$ & $4.3 \times 10^{-2}$ \\
\hline 1 & $1.4 \times 10^{-5}$ & $1.6 \times 10^{-4}$ & $1.0 \times 10^{-6}$ & $2.1 \times 10^{-5}$ \\
\hline 2 & $5.3 \times 10^{-8}$ & $6.2 \times 10^{-7}$ & $3.2 \times 10^{-10}$ & $6.4 \times 10^{-9}$ \\
\hline 3 & $2.0 \times 10^{-10}$ & $2.4 \times 10^{-9}$ & $1.0 \times 10^{-13}$ & $1.9 \times 10^{-12}$ \\
\hline
\end{tabular}

TABLE 7. Sloan method, orthogonal projection, $k(s, t)=\exp (s t)$.

\begin{tabular}{|c|c|c|c|c|}
\hline$k$ & $\left|\lambda^{R}-\lambda_{n}^{(k)}\right|$ & $\left\|\phi^{R}-\phi_{n}^{(k)}\right\|$ & $\left|\lambda^{R}-\mu_{n}^{(k)}\right|$ & $\left\|\phi^{R}-\psi_{n}^{(k)}\right\|$ \\
\hline 0 & $2.4 \times 10^{-3}$ & $9.3 \times 10^{-4}$ & $2.4 \times 10^{-3}$ & $9.3 \times 10^{-4}$ \\
\hline 1 & $1.4 \times 10^{-5}$ & $3.5 \times 10^{-6}$ & $1.0 \times 10^{-6}$ & $8.5 \times 10^{-7}$ \\
\hline 2 & $5.3 \times 10^{-8}$ & $1.3 \times 10^{-8}$ & $3.1 \times 10^{-10}$ & $2.4 \times 10^{-10}$ \\
\hline 3 & $2.0 \times 10^{-10}$ & $5.1 \times 10^{-11}$ & $9.0 \times 10^{-14}$ & $7.2 \times 10^{-14}$ \\
\hline
\end{tabular}

Interpolatory projection. Collocation at Gauss 2 points We choose $X_{n}$ to be the space of piecewise linear functions $(r=2)$ with respect to the equidistant partition (6.1). The collocation points are Gauss 2 points

$$
\tau_{k 1}^{n}=\frac{2 k-1-1 / \sqrt{3}}{2 n}, \quad \tau_{k 2}^{n}=\frac{2 k-1+1 / \sqrt{3}}{2 n}, \quad k=1, \ldots, n .
$$

The projection $\pi_{n}: C[0,1] \rightarrow X_{n}$ is the interpolatory projection. As before we fix $n=2$ and $R=512$. 
TABLE 8. New method, collocation at Gauss 2 points, $k(s, t)=\exp (s t)$.

\begin{tabular}{|c|c|c|c|c|}
\hline$k$ & $\left|\lambda^{R}-\lambda_{n}^{(k)}\right|$ & $\left\|\phi^{R}-\phi_{n}^{(k)}\right\|$ & $\left|\lambda^{R}-\mu_{n}^{(k)}\right|$ & $\left\|\phi^{R}-\psi_{n}^{(k)}\right\|$ \\
\hline 0 & $2.3 \times 10^{-8}$ & $5.1 \times 10^{-6}$ & $2.3 \times 10^{-8}$ & $5.1 \times 10^{-6}$ \\
\hline 1 & $1.0 \times 10^{-12}$ & $2.1 \times 10^{-9}$ & $*$ & $9.5 \times 10^{-13}$ \\
\hline 2 & $*$ & $7.4 \times 10^{-11}$ & $*$ & $*$ \\
\hline
\end{tabular}

TABLE 9. Galerkin method, collocation at Gauss 2 points, $k(s, t)=\exp (s t)$.

\begin{tabular}{|c|c|c|c|c|}
\hline$k$ & $\left|\lambda^{R}-\lambda_{n}^{(k)}\right|$ & $\left\|\phi^{R}-\phi_{n}^{(k)}\right\|$ & $\left|\lambda^{R}-\mu_{n}^{(k)}\right|$ & $\left\|\phi^{R}-\psi_{n}^{(k)}\right\|$ \\
\hline 0 & $4.2 \times 10^{-5}$ & $7.5 \times 10^{-3}$ & $4.2 \times 10^{-5}$ & $7.5 \times 10^{-3}$ \\
\hline 1 & $4.2 \times 10^{-5}$ & $2.7 \times 10^{-5}$ & $2.2 \times 10^{-8}$ & $1.1 \times 10^{-7}$ \\
\hline 2 & $2.2 \times 10^{-8}$ & $1.1 \times 10^{-7}$ & $5.5 \times 10^{-13}$ & $4.6 \times 10^{-12}$ \\
\hline 3 & $6.2 \times 10^{-10}$ & $2.1 \times 10^{-10}$ & $*$ & $*$ \\
\hline
\end{tabular}

TABLE 10. Projection method, collocation at Gauss 2 points, $k(s, t)=\exp (s t)$.

\begin{tabular}{|c|c|c|c|c|}
\hline$k$ & $\left|\lambda^{R}-\lambda_{n}^{(k)}\right|$ & $\left\|\phi^{R}-\phi_{n}^{(k)}\right\|$ & $\left|\lambda^{R}-\mu_{n}^{(k)}\right|$ & $\left\|\phi^{R}-\psi_{n}^{(k)}\right\|$ \\
\hline 0 & $4.2 \times 10^{-5}$ & $5.5 \times 10^{-3}$ & $4.2 \times 10^{-5}$ & $5.5 \times 10^{-3}$ \\
\hline 1 & $2.2 \times 10^{-8}$ & $2.0 \times 10^{-6}$ & $6.2 \times 10^{-10}$ & $7.4 \times 10^{-8}$ \\
\hline 2 & $8.7 \times 10^{-12}$ & $4.9 \times 10^{-9}$ & $*$ & $8.4 \times 10^{-13}$ \\
\hline 3 & $7.5 \times 10^{-14}$ & $1.0 \times 10^{-11}$ & $*$ & $*$ \\
\hline
\end{tabular}

TABLE 11. Sloan method, collocation at Gauss 2 points, $k(s, t)=\exp (s t)$.

\begin{tabular}{|c|c|c|c|c|}
\hline$k$ & $\left|\lambda^{R}-\lambda_{n}^{(k)}\right|$ & $\left\|\phi^{R}-\phi_{n}^{(k)}\right\|$ & $\left|\lambda^{R}-\mu_{n}^{(k)}\right|$ & $\left\|\phi^{R}-\psi_{n}^{(k)}\right\|$ \\
\hline 0 & $4.2 \times 10^{-5}$ & $2.7 \times 10^{-5}$ & $4.2 \times 10^{-5}$ & $2.7 \times 10^{-5}$ \\
\hline 1 & $2.2 \times 10^{-8}$ & $1.1 \times 10^{-8}$ & $6.2 \times 10^{-10}$ & $3.1 \times 10^{-10}$ \\
\hline 2 & $8.7 \times 10^{-12}$ & $2.0 \times 10^{-10}$ & $*$ & $3.1 \times 10^{-14}$ \\
\hline 3 & $7.6 \times 10^{-14}$ & $1.1 \times 10^{-13}$ & $*$ & $*$ \\
\hline
\end{tabular}

We choose

$$
w_{p}^{(R)}=\frac{1}{R}, \quad \xi_{p}^{(R)}= \begin{cases}(p-1 / \sqrt{3}) / R, & \text { if } p \text { is odd } \\ (p-1+1 / \sqrt{3}) / R, & \text { if } p \text { is even. }\end{cases}
$$

Green's kernel Tables 12-13 illustrate that the new method is superior to the Galerkin method even when the kernel is only continuous. Here we choose $n=8$ and $m=256$ so that $R=512$. We approximate the 10th eigenvalue $\lambda^{R}$ of $T_{R}$ and a corresponding eigenvector. The corresponding exact eigenvalue $\lambda$ of $T$ is given by $1 / 100 \pi^{2}$. Note that since $\left|\lambda-\lambda^{R}\right|=3.4 \times 10^{-7}$, we can expect to achieve at the most an accuracy of $10^{-7}$ by the iterative methods. 
TABLE 12. New method, collocation at Gauss 2 points, Green's kernel.

\begin{tabular}{|c|c|c|c|c|}
\hline$k$ & $\left|\lambda^{R}-\lambda_{n}^{(k)}\right|$ & $\left\|\phi^{R}-\phi_{n}^{(k)}\right\|$ & $\left|\lambda^{R}-\mu_{n}^{(k)}\right|$ & $\left\|\phi^{R}-\psi_{n}^{(k)}\right\|$ \\
\hline 0 & $7.2 \times 10^{-5}$ & $1.8 \times 10^{-4}$ & $7.2 \times 10^{-5}$ & $1.8 \times 10^{-4}$ \\
\hline 1 & $2.5 \times 10^{-5}$ & $5.0 \times 10^{-5}$ & $4.1 \times 10^{-6}$ & $1.6 \times 10^{-5}$ \\
\hline 2 & $6.6 \times 10^{-6}$ & $4.2 \times 10^{-5}$ & $1.9 \times 10^{-9}$ & $5.2 \times 10^{-7}$ \\
\hline 3 & $3.3 \times 10^{-6}$ & $1.5 \times 10^{-5}$ & $*$ & $*$ \\
\hline 4 & $1.5 \times 10^{-6}$ & $3.7 \times 10^{-6}$ & $*$ & $*$ \\
\hline 5 & $4.6 \times 10^{-7}$ & $1.6 \times 10^{-6}$ & $*$ & $*$ \\
\hline
\end{tabular}

TABLE 13. Galerkin method, collocation at Gauss 2 points, Green's kernel.

\begin{tabular}{|c|c|c|c|c|}
\hline$k$ & $\left|\lambda^{R}-\lambda_{n}^{(k)}\right|$ & $\left\|\phi^{R}-\phi_{n}^{(k)}\right\|$ & $\left|\lambda^{R}-\mu_{n}^{(k)}\right|$ & $\left\|\phi^{R}-\psi_{n}^{(k)}\right\|$ \\
\hline 0 & $1.0 \times 10^{-4}$ & $3.5 \times 10^{-4}$ & $1.0 \times 10^{-4}$ & $3.4 \times 10^{-4}$ \\
\hline 1 & $1.0 \times 10^{-5}$ & $9.8 \times 10^{-5}$ & $2.8 \times 10^{-5}$ & $5.6 \times 10^{-5}$ \\
\hline 2 & $2.8 \times 10^{-5}$ & $5.6 \times 10^{-5}$ & $2.8 \times 10^{-5}$ & $8.3 \times 10^{-5}$ \\
\hline 3 & $4.6 \times 10^{-5}$ & $2.8 \times 10^{-5}$ & $1.5 \times 10^{-5}$ & $1.7 \times 10^{-5}$ \\
\hline 4 & $2.5 \times 10^{-5}$ & $3.2 \times 10^{-5}$ & $1.6 \times 10^{-6}$ & $3.8 \times 10^{-6}$ \\
\hline 5 & $1.2 \times 10^{-5}$ & $1.7 \times 10^{-5}$ & $1.1 \times 10^{-6}$ & $2.7 \times 10^{-6}$ \\
\hline
\end{tabular}

REMARK. It may be seen from the above results that the iterates in the elementary as well as the double iteration using the new method converge faster than the corresponding iterates obtained using the Galerkin, projection or Sloan methods.

\section{References}

[1] M. Ahues, A. Largillier and B. V. Limaye, Spectral computations for bounded operators (Chapman and Hall/CRC, New York, 2001).

[2] K. Atkinson and A. Bogomolny, "The discrete Galerkin method for integral equations", Math. Comp. 48 (1987).

[3] F. Chatelin, Spectral approximation of linear operators (Academic Press, New York, 1983).

[4] C. de Boor, "A bound on the $L_{\infty}$ norm of $L_{2}$-approximation by splines in terms of a global mesh ratio", Math. Comp. 30 (1976) 765-771.

[5] C. de Boor and B. Swartz, "Collocation at Gaussian points", SIAM J. Numer. Anal. 10 (1973) $582-606$.

[6] L. N. Deshpande and B. V. Limaye, "A fixed point technique to refine a simple approximate eigenvalue and corresponding eigenvector", Numer. Funct. Anal. Optim. 10 (1989) 909-921.

[7] J. Douglas, Jr., T. Dupont and L. Wahlbin, "Optimal $L_{\infty}$ error estimates for Galerkin approximations to solutions of two point boundary value problems", Math. Comp. 29 (1975) 475-483.

[8] R. P. Kulkarni, “A superconvergence result for solutions of compact operator equations", Bull. Austral. Math. Soc. 68 (2003) 517-528. 
[9] R. P. Kulkarni, “A new superconvergent projection method for approximate solutions of eigenvalue problems", Numer. Funct. Anal. Optim. 24 (2003) 75-84.

[10] R. P. Kulkarni and B. V. Limaye, "Solution of a Schrodinger equation by iterative refinement", $J$. Austral. Math. Soc. Ser. B 32 (1990) 115-132.

[11] J. E. Osborn, "Spectral approximation for compact operators", Math. Comp. 29 (1975) 712-725.

[12] G. R. Richter, "Superconvergence of piecewise polynomial Galerkin approximations for Fredholm integral equations of the second kind", Numer. Math. 31 (1978) 63-70. 